\title{
A systematic review of the intergenerational aspects and the diverse genetic profiles of Huntington's disease
}

\author{
L.A. Agostinho ${ }^{1,2}$, S.R. dos Santos $^{1}$, R.M.P. Alvarenga ${ }^{2}$ and C.L.A. Paiva ${ }^{1,2}$ \\ ${ }^{1}$ Departamento de Genética e Biologia Molecular, Instituto Biomédico, \\ Rio de Janeiro, RJ, Brasil \\ ${ }^{2}$ Programa de Pós-Graduação em Neurologia, \\ Universidade Federal do Estado do Rio de Janeiro, Rio de Janeiro, RJ, Brasil \\ Corresponding author: C.L.A. Paiva \\ E-mail: clapaiva1@gmail.com
}

Genet. Mol. Res. 12 (2): 1974-1981 (2013)

Received December 13, 2012

Accepted May 15, 2013

Published June 13, 2013

DOI http://dx.doi.org/10.4238/2013.June.13.6

\begin{abstract}
Huntington's disease (HD) is a rare progressive and fatal neurogenetic degenerative disease, characterized by movement and personality disorders and by progressive dementia. Its prevalence varies by ethnic origin and different genetic profiles predisposing individuals to HD in each population. The prevalence of HD is 5-10 per 100,000 individuals in Caucasian populations of North America and Western Europe. It is an autosomal dominant disease associated with the expansion of CAG-type repetitive DNA sequences in the HTT gene. This gene, located on the short arm of chromosome 4, encodes the protein huntingtin. In this study, we reviewed 17 articles about HD that report data from 2400 affected individuals from various countries around the world, including Venezuela, China, Croatia, Turkey, Germany, Italy, Brazil, Spain, Taiwan, India, the Netherlands, Russia, and the USA, with a focus on genetic profiles and intergenerational expansions or contractions of expanded alleles responsible for causing HD. We discuss the genetic characteristics of HD in different
\end{abstract}


populations and any atypical cases reported in these studies.

Key words: Huntington's disease; CAG repeats; Intergenerational aspects

\section{INTRODUCTION}

Huntington's disease (HD) is a rare progressive and fatal neurodegenerative disease, characterized by a lack of motor coordination of voluntary and involuntary muscles, known as choreic movement, personality disorders, and progressive dementia (Chandler et al., 1960). The genetic profiles of HD and the intergenerational aspects of expanded allele transmission vary across different regions of the world. The prevalence of $\mathrm{HD}$ varies with ethnic origin, with Caucasian populations of North America and Western Europe having 5-10 subjects affected by HD per 100,000 people (Lima et al., 2000; Hormozian et al., 2004; Gil and Rego, 2008). Together with literature reports of atypical cases of $\mathrm{HD}$, the above-mentioned variations indicate that there is a need for a systematic review of the HD literature. To this end, this review aggregates information about the genetic profiles of HD in different populations and atypical cases.

$\mathrm{HD}$ is caused by a mutation triggered by variations in the number of CAG repeats in unstable DNA regions of the HTT gene, which is located on the short arm of chromosome 4 (4p16.3). The mutable region is located in the first exon of $H T T$ and encodes a polyglutamine $\mathrm{N}$-terminal tail of the encoded protein, huntingtin (Wexler et al., 2004). Alleles that bear 27 or fewer copies of the CAG repeat result in a normal phenotype. Intermediary alleles, which have 27-35 CAG repeats, are unstable and can be transmitted as an expanded allele to offspring; nevertheless, carriers of the intermediary allele type also have a normal phenotype. However, intermediary alleles get expanded mainly during male gametogenesis, which undergoes a greater number of cycles of division and duplication of genetic material than oogenesis. Hence, there is a greater probability of an error occurring in DNA replication during spermatogenesis, which increases the risk of mutated alleles being inherited from the father (Wheeler et al., 2007).

Alleles with 36-39 CAG units have reduced penetrance and generate both a normal phenotype as well as some rare cases of HD. Alleles with $>39$ CAG copies show complete penetrance and inevitably cause, at some stage of life, the HD phenotype.

Classical studies examining the geographical distribution of HD, along with several epidemiological studies, suggest that the alleles responsible for causing HD originated from a single mutation in a common ancestor from Western Europe, and this mutation then spread to other regions of the world as a result of migration. Different haplotypes that include the CCG repeat region, which is adjacent to the CAG region, were identified in different populations and confirm the existence of mutations that had independent and distinct origins from that of a single common HD ancestor (García-Planells et al., 2005).

In patients with $\mathrm{HD}$, the onset of clinical symptoms usually starts between the ages of 35-55, although the disease can manifest after the age of 80, or much earlier, in teenagers or children, because of the phenomenon of anticipation, which occurs in $20 \%$ of cases of HD. Approximately $10 \%$ of patients with HD have onset of clinical manifestations before the age of 20 , and $5 \%$ before the age of 14 . If the expanded allele transmitted to the offspring is of maternal origin, an expansion of $>20$ units is very rare (Andrew et al., 1993; Nahhas et al., 2005). 
The incidence of genetically confirmed cases of HD in individuals who have no family history of HD and who would therefore be the first confirmed case in the family is greater than $8 \%$. These cases are caused by new, sporadic expansions of intermediary alleles. Such unstable alleles favor the emergence of mutated alleles in offspring, which leads to HD (Nahhas et al., 2005).

From the onset of initial symptoms, HD will progress to death within 15-20 years (Ho et al., 2001). Variability in clinical signs and symptoms of the disease often requires a precise molecular investigation at the genetic level to enable final diagnosis (Stevanin et al., 2003).

HD is an autosomal dominant genetic disease. Each child of an affected parent has a $50 \%$ chance of developing the disease; therefore, only one allele inherited from the mother, or from the father, is able to cause the disease in the offspring. However, although very rare, when in the homozygous state, the 2 expanded alleles result in increased physical deterioration of HD-affected individuals when compared to affected individuals bearing only one expanded allele. Studies further suggest that homozygosity in the expanded alleles does not lead to an earlier onset of the disease, but changes the severity of HD symptoms and their progression (Squitieri et al., 1994).

\section{MATERIAL AND METHODS}

A search was conducted on the Internet using databases related to the health sciences, such as Pubmed/Medline, Scielo, and Bireme. Articles were selected from these 3 databases. The key words used were "Huntington's disease" and "CAG repeats". Advanced search functions were used to restrict the search to articles published from 2000-2012.

\section{RESULTS}

We identified 616 scientific articles published from 2000-2012, and 41 of them were selected according to the chosen theme. Of the 41 articles, 17 were chosen because they reported detailed molecular investigations of HD, intergenerational analysis of expanded alleles in different regions of the world, and atypical cases of the disease (Table 1). The 2381 individuals investigated who were described in these papers are the subject of discussion in this systematic review. Of the selected articles, 8 reported anticipation in HD-affected patients, and 5 articles discussed cases of juvenile HD primarily caused by paternal inheritance.

Studies conducted in Venezuela on 112 individuals with HD confirmed the high instability of alleles transmitted through the father, as indicated by the higher frequency of juvenile HD with paternal inheritance (Wheeler et al., 2007). However, 2 studies of juvenile HD detected no significant difference in the frequency of HD between the paternal and maternal routes of inheritance (Nahhas et al., 2005).

In 2005, a molecular investigation was conducted on 2 individuals who had inherited HD with anticipation from the mother. She carried an allele with 70 CAG repeats, and had transmitted an allele containing 130 CAG copies (Nahhas et al., 2005).

Few articles have reported data on Brazilian HD patients. These articles reported investigations of CAG repeats-containing alleles at the molecular level (Lima et al., 2000; Raskin et al., 2000; Agostinho et al., 2012). One of these papers correlated genetic character- 
Huntington's disease: a systematic review

1977

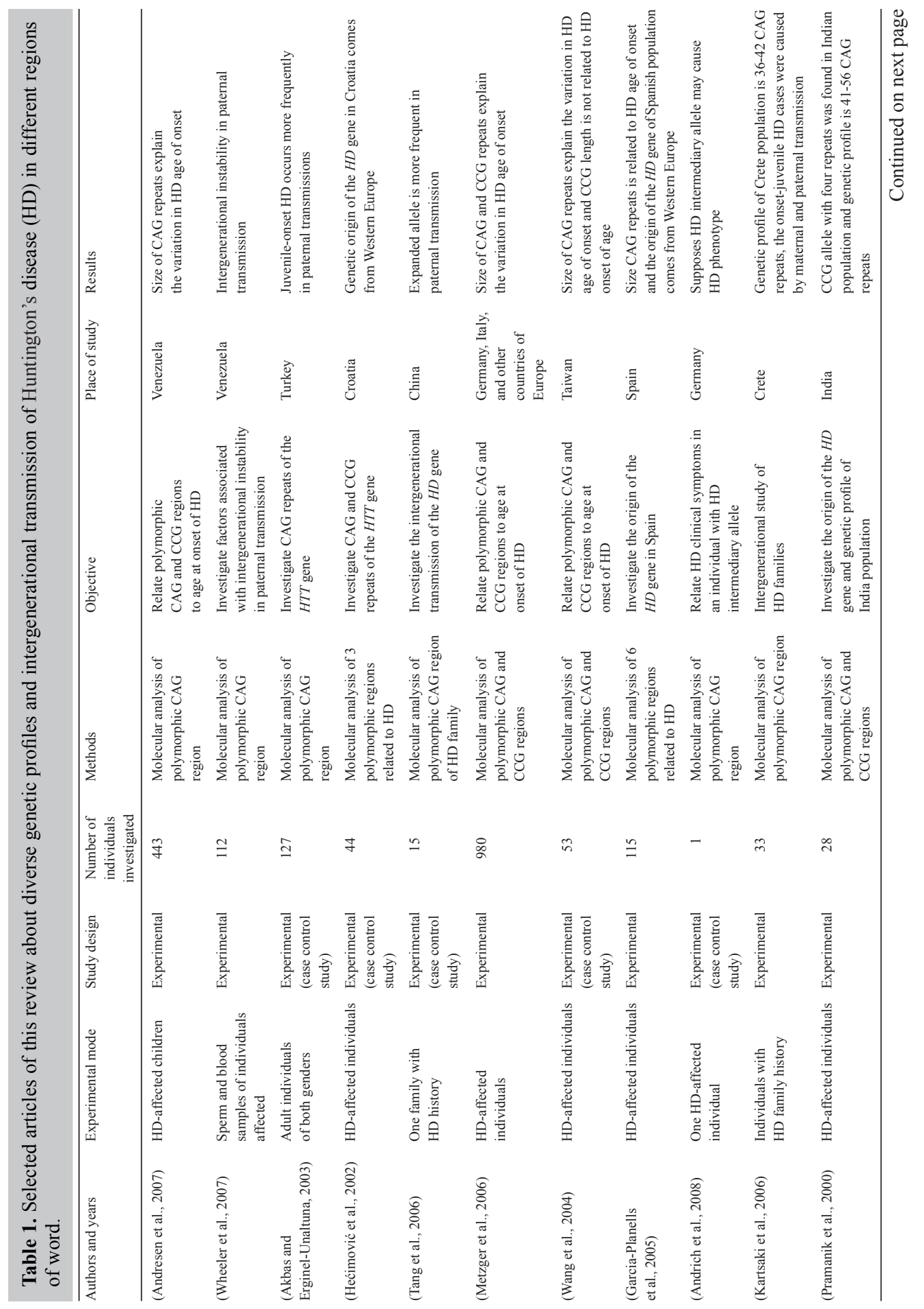

Genetics and Molecular Research 12 (2): 1974-1981 (2013)

CFUNPEC-RP www.funpecrp.com.br 
L.A. Agostinho et al.

1978

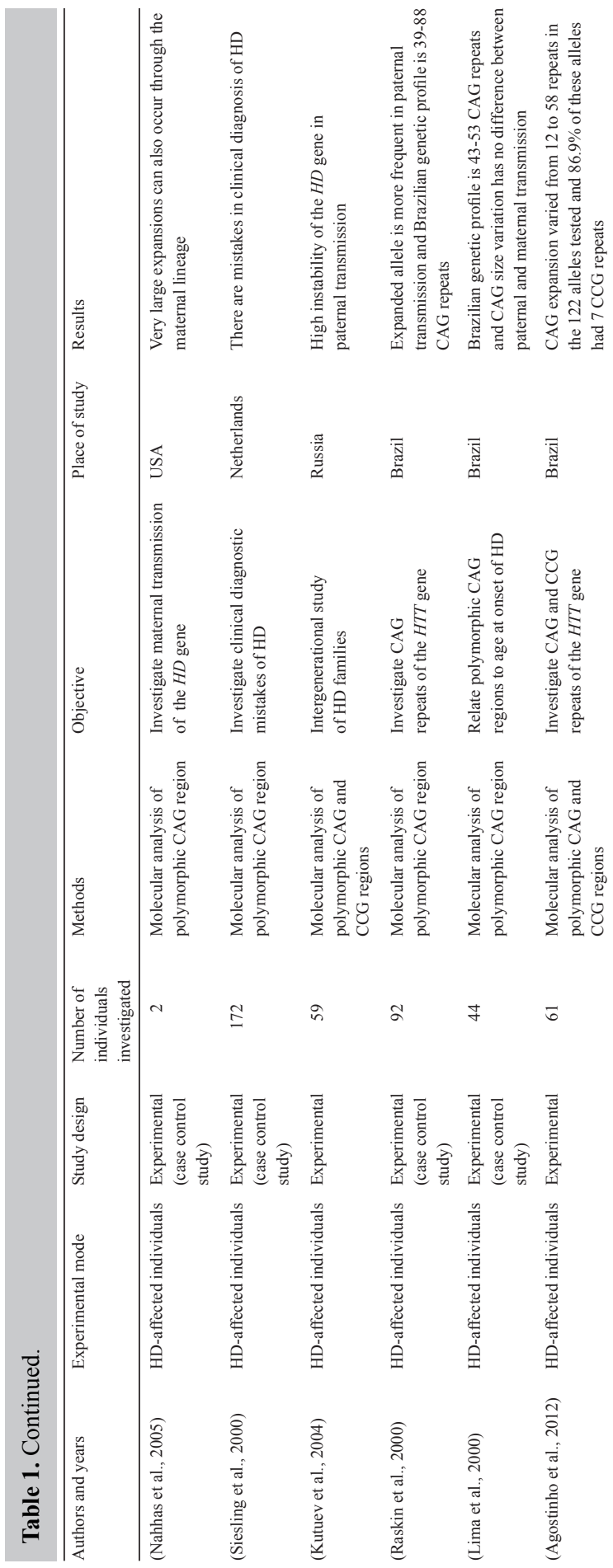

Genetics and Molecular Research 12 (2): 1974-1981 (2013) 
istics of HD with the age at onset of the clinical symptoms (Lima et al., 2000). A case-control study investigated $84 \mathrm{HD}$-affected individuals. In another study, the shortest HD allele identified contained $39 \mathrm{CAG}$ repeats. An inverse correlation between the onset of clinical manifestations and the size of the CAG-repeat regions was also observed (Raskin et al., 2000). Furthermore, in subjects showing trinucleotide expansions, these expansions were longer when the mutated allele was inherited from the father than when it was inherited from the mother (Raskin et al., 2000).

In 2012, a molecular study in Brazil reported that the length of the CAG expansion varied from $12-58$ repeats in 122 alleles tested. In the heterozygous subjects $(\mathrm{N}=46)$, who carried at least one expanded allele each, the normal alleles contained 12-26 CAG repeats with a mean of $16 \pm 3.3$ and a median of 15 . The shortest expanded allele that caused an HD phenotype contained $37 \mathrm{CAG}$ repeats, and the longest contained 58 repeats. None of subjects was homozygous for an expanded CAG allele (Agostinho et al., 2012).

In the same study, repeats of 7 CCG trinucleotides were found in $78.7 \%$ of the sample. The CCG alleles, linked to the expanded CAG alleles had 6, 7, 8, or 10 CCG repeats (mean $7.1 \pm 0.7$ ), and $86.9 \%$ of these alleles had 7 CCG repeats. This was the first investigation of the polymorphic CCG region in Brazil (Agostinho et al., 2012).

In Turkey, a study of 127 individuals showed that the shortest HD-causing allele contained $38 \mathrm{CAG}$ copies, and the longest allele contained $78 \mathrm{CAG}$ repeats (Akbas and ErginelUnaltuna, 2003).

Another survey conducted on the Crete island, Greece, studying HD in 33 individuals, reported that the shortest HD-causing allele contained $36 \mathrm{CAG}$ copies and the longest allele had 42 CAG repeats (Kartsaki et al., 2006). In India, the shortest HD allele identified contained 41 CAG repeats and the longest allele, 56 CAG repeats (Pramanik et al., 2000).

Furthermore, it was in India where, for the first time, 4 CCG repeats were found adjacent to the $\mathrm{CAG}$ region in a subject with $\mathrm{HD}$. The $\mathrm{CCG}$ region next to the $\mathrm{CAG}$ region has also been the target of many molecular studies. These studies are useful for confirming the results of molecular diagnosis or for the genetic study of the ancestral origin of causative HD alleles. Most of the articles that have analyzed the CCG regions reported that the genetic origin of the expanded HD alleles was Western Europe (Hećimović et al., 2002; García-Planells et al., 2005).

An experimental study with 53 patients suggested that the size of the CCG repeats is not a determining factor for the age of onset of HD (Wang et al., 2004), a finding that was confirmed by Agostinho et al. (2012). On the other hand, a study with 980 individuals in European countries provided strong evidence for an association of the size of the CAG and CCG repeats with age at onset of HD (Metzger et al., 2006).

Among the individuals studied in Brazil, the smallest allele causing onset of HD contained 37 CAG repeats and the longest allele had 88 CAG repeats (Lima et al., 2000; Raskin et al., 2000; Agostinho et al., 2012). In 2008, an atypical case was reported from Germany of a patient who was affected by HD even though the disease allele contained only 34 copies of the CAG repeat (Andrich et al., 2008).

\section{DISCUSSION}

HD is a disease of adults. Juvenile cases are rare (only approximately $10 \%$ of total cases), and the majority of the affected individuals inherit expanded alleles from their fathers 
(Nahhas et al., 2005). However, one study showed no significant difference in the number of cases of juvenile HD between paternally or maternally inherited HD (Lima et al., 2000). The CAG repeat in a maternal allele usually does not expand to more than 20 copies (Nahhas et al., 2005; Kenney et al., 2007); however, a report in 2005 showed that an affected mother had transmitted a double-size allele to her daughter (Nahhas et al., 2005).

Two case reports in the literature have identified individuals who carried intermediary alleles and who were clinically diagnosed as being affected by HD. In one article, an individual who had an allele with 29 CAG repeats was diagnosed with HD by post-mortem autopsy (Andresen et al., 2007). Another article has reported that an individual who presented cortical atrophy, disorder of movement, and cognitive impairment carried an allele with only 34 copies of the CAG repeat (Andrich et al., 2008). However, neither study mentions any attempts by investigators to diagnose a Huntington-like disease.

Among the studies on HD, where differential diagnosis for other similar diseases was performed, the smallest number of CAG units associated with the HD phenotype was 37, identified in a patient of European descent. The absence of the clinical symptoms of HD has not yet been documented in any individual carrying an allele containing $>39$ CAG repeats.

Alleles with 7 CCG units were observed in $95 \%$ of cases. This finding might mean that those alleles had a genetic origin in Western Europe, the presumed location of a founder effect for HD. On the other hand, alleles containing 10 CCG units may have been the result of independent mutations that recently originated from other geographical regions. Usually these CCG alleles possess 7 or 10 repeats, with the exception of a case, reported by Pramanik et al. (2000), in which 4 CCG units were identified.

In conclusion, a molecular test that determines the length of a CAG repeat is essential because it can provide a definite diagnosis of HD. It is important to note that only about $40 \%$ of the carriers of the reduced penetrance allele may show the HD phenotype. Furthermore, during clinical investigation, the following procedures must be taken into account: differential diagnosis for atypical cases must be performed, along with paternity tests to confirm the origin of the disease alleles, mainly for new cases in the family.

In addition, it is difficult to define the smallest size of expanded CAG repeats responsible for the HD phenotype because there are many differences among individuals and populations. A definite clinical diagnosis often requires caution because the clinical presentation of signs and symptoms of HD is variable.

\section{ACKNOWLEDGMENTS}

Reseach supported by CAPES/PROAP, FINEP, PROPGNEURO, and Departamento de Genética e Biologia Molecular, UNIRIO.

\section{REFERENCES}

Agostinho L de A, Rocha CF, Medina-Acosta E, Barboza HN, et al. (2012). Haplotype analysis of the CAG and CCG repeats in 21 Brazilian families with Huntington's disease. J. Hum. Genet. 57: 796-803.

Akbas F and Erginel-Unaltuna N (2003). DNA testing for Huntington disease in the Turkish population. Eur. Neurol. 50: 20-24.

Andresen JM, Gayán J, Cherny SS, Brocklebank D, et al. (2007). Replication of twelve association studies for Huntington's disease residual age of onset in large Venezuelan kindreds. J. Med. Genet. 44: 44-50. 
Andrew SE, Goldberg YP, Kremer B, Telenius H, et al. (1993). The relationship between trinucleotide (CAG) repeat length and clinical features of Huntington's disease. Nat. Genet. 4: 398-403.

Andrich J, Arning L, Wieczorek S, Kraus PH, et al. (2008). Huntington's disease as caused by 34 CAG repeats. Mov. Disord. 23: 879-881.

Chandler JH, Reed TE and Dejong RN (1960). Huntington's chorea in Michigan. III. Clinical observations. Neurology 10: $148-153$.

García-Planells J, Burguera JA, Solís P, Millán JM, et al. (2005). Ancient origin of the CAG expansion causing Huntington disease in a Spanish population. Hum. Mutat. 25: 453-459.

Gil JM and Rego AC (2008). Mechanisms of neurodegeneration in Huntington's disease. Eur. J. Neurosci. 27: 2803-2820.

Hećimović S, Klepac N, Vlasić J, Vojta A, et al. (2002). Genetic background of Huntington disease in Croatia: Molecular analysis of CAG, CCG, and Delta2642 (E2642del) polymorphisms. Hum. Mutat. 20: 233.

Ho LW, Carmichael J, Swartz J, Wyttenbach A, et al. (2001). The molecular biology of Huntington's disease. Psychol. Med. 31: 3-14.

Hormozian F, Houshmand M, Sanati MH, Ghiasvand R, et al. (2004). Molecular analysis of the (CAG)n repeat causing Huntington's disease in 34 Iranian families. Indian J. Hum. Genet. 10: 53-58.

Kartsaki E, Spanaki C, Tzagournissakis M, Petsakou A, et al. (2006). Late-onset and typical Huntington disease families from Crete have distinct genetic origins. Int. J. Mol. Med. 17: 335-346.

Kenney C, Powell S and Jankovic J (2007). Autopsy-proven Huntington's disease with 29 trinucleotide repeats. Mov. Disord. 22: 127-130.

Kutuev IA, Khusainova RI, Khidiyatova IM, Magzhanov RV, et al. (2004). Analysis of the IT15 gene in Huntington's disease families. Russian J. Genet. 40: 919-925.

Lima E Silva TC, Serra HG, Bertuzzo CS and Lopes-Cendes I (2000). Molecular diagnosis of Huntington disease in Brazilian patients. Arq. Neuropsiquiatr. 58: 11-17.

Metzger S, Bauer P, Tomiuk J, Laccone F, et al. (2006). Genetic analysis of candidate genes modifying the age-at-onset in Huntington's disease. Hum. Genet. 120: 285-292.

Nahhas FA, Garbern J, Krajewski KM, Roa BB, et al. (2005). Juvenile onset Huntington disease resulting from a very large maternal expansion. Am. J. Med. Genet. A 137A: 328-331.

Pramanik S, Basu P, Gangopadhaya PK, Sinha KK, et al. (2000). Analysis of CAG and CCG repeats in Huntingtin gene among HD patients and normal populations of India. Eur. J. Hum. Genet. 8: 678-682.

Raskin S, Allan N, Teive HA, Cardoso F, et al. (2000). Huntington disease: DNA analysis in Brazilian population. Arq. Neuropsiquiatr. 58: 977-985.

Siesling S, Vegter-van de Vlis M, Losekoot M, Belfroid RD, et al. (2000). Family history and DNA analysis in patients with suspected Huntington's disease. J. Neurol. Neurosurg. Psychiatry 69: 54-59.

Squitieri F, Andrew SE, Goldberg YP, Kremer B, et al. (1994). DNA haplotype analysis of Huntington disease reveals clues to the origins and mechanisms of CAG expansion and reasons for geographic variations of prevalence. Hum. Mol. Genet. 3: 2103-2114.

Stevanin G, Fujigasaki H, Lebre AS, Camuzat A, et al. (2003). Huntington's disease-like phenotype due to trinucleotide repeat expansions in the TBP and JPH3 genes. Brain 126: 1599-1603.

Tang Y, Wang Y, Yang P, Liu Y, et al. (2006). Intergeneration CAG expansion and contraction in a Chinese HD family. Am. J. Med. Genet. B Neuropsychiatr. Genet. 141B: 242-244.

Wang CK, Wu YR, Hwu WL, Chen CM, et al. (2004). DNA haplotype analysis of CAG repeat in Taiwanese Huntington's disease patients. Eur. Neurol. 52: 96-100.

Wexler NS, Lorimer J, Porter J, Gomez F, et al. (2004). Venezuelan kindreds reveal that genetic and environmental factors modulate Huntington's disease age of onset. Proc. Natl. Acad. Sci. U. S. A. 101: 3498-3503.

Wheeler VC, Persichetti F, McNeil SM, Mysore JS, et al. (2007). Factors associated with HD CAG repeat instability in Huntington disease. J. Med. Genet. 44: 695-701. 